\title{
FATORES DE RISCO E PROTEÇÃO CONTRA DOENÇAS CRÔNICAS NÃO TRANSMISSÍVEIS ENTRE ADOLESCENTES
}

\section{Risk and protective factors for noncommunicable diseases among adolescents \\ Factores de riesgo y protección para las enfermedades crónicas no transmisibles entre adolescentes}

Keise Adrielle Santos Pereira

Universidade Estadual da Região Tocantina do Maranhão - UEMASUL - Imperatriz (MA) - Brasil

Sheila Elke Araújo Nunes

Universidade Estadual da Região Tocantina do Maranhão - UEMASUL - Imperatriz (MA) - Brasil

Márcia Guelma Santos Belfort

Unidade de Ensino Superior do Sul do Maranhão - UNISULMA - Imperatriz (MA) - Brasil

Ray Sousa Alves Miranda

Universidade Estadual da Região Tocantina do Maranhão - UEMASUL - Imperatriz (MA) - Brasil

\section{Aline Dias Horas}

Universidade Estadual da Região Tocantina do Maranhão - UEMASUL - Imperatriz (MA) - Brasil

Joaquim Paulo de Almeida Júnior

Universidade Estadual da Região Tocantina do Maranhão - UEMASUL - Imperatriz (MA) - Brasil

\section{Antonia Marcia Meireles}

Secretaria Municipal de Educação - SEMED - Imperatriz (MA) - Brasil

Valdina dos Santos Aguiar

Secretaria Municipal de Educação - SEMED - Imperatriz (MA) - Brasil

\section{RESUMO}

Objetivo: Conhecer a prevalência dos fatores de risco para doenças crônicas não transmissíveis (DCNT) entre adolescentes assistidos pelo programa municipal Saúde e Prevenção na Escola (SPE). Métodos: Estudo de corte transversal realizado com escolares de 10 a 17 anos pertencentes a seis escolas do município de Imperatriz, Maranhão, Brasil, entre outubro de 2014 e abril de 2015. Medidas antropométricas foram coletadas na própria escola e as variáveis associadas aos hábitos alimentares e estilo de vida foram investigadas por meio de questionário autoaplicável, sendo comparados os dados entre os sexos através do teste Qui-quadrado. Resultados: Foram avaliados 473 estudantes entre 10 e 17 anos. O sobrepeso e a obesidade ocorrem em 13,3\% (63) da amostra. Alimentos como arroz, feijão e frutas têm consumo associado a fatores de proteção e apresentaram maiores proporções de consumo entre o sexo feminino $(p=0,001)$, enquanto a prática de atividade física prevalece entre o sexo masculino $(\mathrm{p}=0,0001)$. A exposição ao cigarro, drogas ilícitas e álcool não se distingue entre os sexos. Conclusão: A população estudada apresenta distintos padrões de exposição a fatores de risco e proteção, e evidencia a necessidade de ações educativas no ambiente escolar conduzidas pelos profissionais do SPE e demais gestores escolares.

Descritores: Estudantes; Ensino Fundamental e Médio; Alimentação; Estilo de vida. 


\section{ABSTRACT}

Objective: To know the prevalence of risk factors for noncommunicable diseases (NCDs) among adolescents assisted by the municipal program "Health and Prevention at School" (Saúde e Prevenção na Escola-SPE). Method: A cross-sectional study was carried out with students aged 10 to 17 years from six schools located in the municipality of Imperatriz, Maranhão, Brazil, between October 2014 and April 2015. Anthropometric measures were collected at the school and variables related to eating habits and lifestyle were investigated using a self-administered questionnaire. Data were compared between genders using the Chi-squared test. Results: A total of 473 students aged 1017 years were assessed. Overweight and obesity occurred in 13.3\% (63) of the sample. The consumption of foods such as rice, beans and fruits is associated with protective factors. There was a higher consumption of these foods among girls ( $p=0.001)$ and physical activity was prevalent among boys ( $p=0.0001)$. There are no differences in exposure to cigarettes, illicit drugs and alcohol between genders. Conclusion: The study population presents different patterns of exposure to risk and protective factors and there is an evident need for educational actions in the school environment conducted by SPE professionals and other school managers.

Descriptors: Students; Education, Primary and Secondary; Feeding; Lifestyle.

\section{RESUMEN}

Objetivo: Conocer la prevalencia de los factores de riesgo para las enfermedades crónicas no transmisibles (ECNT) entre adolescentes asistidos por el programa municipal Salud y Prevención en la Escuela (SPE). Métodos: Estudio transversal realizado con escolares entre 10 y 17 años de seis escuelas del municipio de Imperatriz, Maranhão, Brasil, entre octubre de 2014 y abril de 2015. Las medidas antropométricas fueron recogidas en la propia escuela y las variables asociadas con los hábitos alimentarios y el estilo de vida fueron investigadas a través de un cuestionario auto-aplicado. Los datos fueron comparados entre los sexos a través de la prueba Chi-Cuadrado. Resultados: Fueron evaluados 473 estudiantes entre 10 y 17 años. El sobrepeso y la obesidad se dieron en el 3,3\% (63) de la muestra. Alimentos como el arroz, los frijoles y las frutas tienen el consumo asociado con factores de protección y presentan mayores proporciones de consumo entre las niñas ( $p=$ $0,001)$ mientras la práctica de actividad física prevalece entre los niños $(p=0,0001)$. No hay diferencia entre los sexos para la exposición al tabaco, las drogas ilícitas y el alcohol. Conclusión: La población estudiada presenta distintos patrones de exposición para los factores de riesgo y protección y es evidente la necesidad de acciones educativas en el ambiente de la escuela que sean conducidas por los profesionales del SPE y demás gestores de las escuelas.

Descriptores: Estudiantes; Educación Primaria y Secundaria; Alimentación; Estilo de vida.

\section{INTRODUÇÃO}

Nas últimas décadas, a exposição a fatores de risco e prevenção à saúde com foco no público adolescente passou a ser um dos temas mais explanados pela literatura ${ }^{(1,2)}$. A importância de estudos com base nessa temática deve-se a vulnerabilidade de crianças e adolescentes durante o período de transição para a vida adulta. Estudos apontam para a probabilidade de adolescentes com sobrepeso e obesidade se tornarem adultos obesos, logo, a obesidade na infância e adolescência é considerada um fator de risco independente no desenvolvimento de várias doenças crônicas não transmissíveis ${ }^{(3,4)}$.

Em função da gravidade das doenças crônicas não transmissíveis (DCNT) e seus impactos nos sistemas de saúde e na sociedade, a Organização das Nações Unidas (ONU), em 2011, debateu compromissos globais sobre o tema, o que culminou em uma declaração política, momento em que os países-membros se comprometem a trabalhar para deter o crescimento das DCNT mediante ações de prevenção de seus principais fatores de risco e empenho pela garantia de uma adequada atenção à saúde ${ }^{(5)}$.

Em maio de 2012, a Assembleia Mundial da Saúde aprovou uma meta global de redução de 25\% da mortalidade prematura por doenças crônicas não transmissíveis entre os anos de 2015 e 2025, destacaram-se também metas de redução de fatores de risco, tais como: diminuição do consumo de tabaco, álcool e sal, sedentarismo e hipertensão arterial; detenção do crescimento da obesidade e do diabetes; tratamento dos pacientes de doenças cardiovasculares e disponibilização de medicamentos para $\mathrm{DCNT}^{(6)}$.

No Plano de Ações Estratégicas para o Enfrentamento das DCNT 2011-2022, traçado pelo Brasil, as metas são coincidentes com as aprovadas na Assembleia Mundial, no que se refere à redução de mortalidade por DCNT. O objetivo do Plano de Enfrentamento é o de promover o desenvolvimento e a implementação de políticas públicas efetivas, baseadas em evidências para a prevenção e o controle das DCNT e seus fatores de risco, além de fortalecer os serviços de saúde voltados às doenças crônicas. O plano aborda os quatro principais grupos de doenças (circulatórias, câncer, respiratórias crônicas e diabetes) e seus fatores de risco em comum que são modificáveis (tabagismo, álcool, inatividade física, alimentação não saudável e obesidade). Uma das metas nacionais propostas pelo plano é reduzir a prevalência de obesidade em adolescentes em vista dos riscos já mencionados $^{(7)}$. 
No Brasil, o Projeto Saúde e Prevenção nas Escolas (SPE) é uma das ações do Programa Saúde na Escola (PSE), que tem a finalidade de contribuir para a formação integral dos estudantes da rede pública de educação básica por meio de ações de prevenção, promoção e atenção à saúde ${ }^{(8)}$.

A partir das questões levantadas nos parágrafos acima, o objeto do presente estudo é conhecer a prevalência dos fatores de risco para doenças crônicas não transmissíveis (DCNT) entre adolescentes assistidos pelo programa municipal Saúde e Prevenção na Escola (SPE), para assim se propor ações de prevenção e promoção da saúde nessa população.

\section{MÉTODOS}

Trata-se de um estudo de corte transversal realizado com escolares de 10 a 17 anos pertencentes a seis escolas do município de Imperatriz, Maranhão, Brasil, entre outubro de 2014 e abril de 2015. Os adolescentes inseridos na pesquisa respeitavam os critérios de inclusão: 1) Estar cursando entre $6^{\circ}$ e $9^{\circ}$ ano, 2) Fazer a assinatura do Termo de Consentimento Livre e Esclarecido (TCLE) (pelo aluno e pelos pais ou responsável), 3) A escola estar cadastrada no Projeto Saúde e Prevenção na Escola (SPE) da Secretária Municipal de Educação e 4) Estar presente na escola no dia da coleta dos dados. Adotaram-se como critérios de exclusão: 1) Estar em algum acompanhamento nutricional e 2) O escolar, mesmo com a autorização do responsável no TCLE, não manifestar vontade em participar da pesquisa.

Para cálculo amostral considerou-se a população de 31.402 escolares matriculados no Ensino Fundamental do município, conforme estimativa do IBGE (2012). Determinou-se nível de confiança de 5\% e intervalo de confiança de $95 \%$ para uma amostra de 380 alunos. Como eventuais perdas são passiveis de ocorrer, ampliou-se a amostra em $25 \%$, incluindo no estudo 481 estudantes. Destes, 5 não apresentaram TCLE assinado pelo responsável e 3 não responderem ao questionário, sendo então excluídos da pesquisa. Portanto, a amostra final foi composta por 473 escolares.

Durante a coleta de dados ocorreram as seguintes etapas: conversa com os gestores para solicitação da pesquisa na escola, com exposição da metodologia da pesquisa, e definição pelos gestores de datas e horários para a coleta dos dados; conversa com os escolares e professores em sala de aula e entrega do TCLE para assinatura; entrega do questionário autoaplicável e exame antropométrico com os escolares. O questionário padronizado foi dividido em sessões, investigaram-se variáveis referentes à: 1) informações demográficas, 2) estilo de vida (hábitos alimentares, prática de exercício físico, uso de drogas e álcool) e 3) registro dos dados do exame antropométrico (peso e altura). Após responderem ao questionário, individualmente, os alunos foram encaminhados a uma sala disponibilizada pela direção da escola para a realização do exame antropométrico.

Os participantes foram pesados e medidos com o uniforme escolar, descalços e sem qualquer acessório, em posição ortostática, com os pés paralelos, braços estendidos ao longo do corpo e cabeça ereta. Para a medida do peso, utilizou-se uma balança eletrônica com precisão de $100 \mathrm{~g}$ e, para mensuração da altura, uma fita métrica com marcações em milímetros, afixada em uma superfície plana, em ângulo de $90^{\circ}$ com o chão.

Para determinar o índice de massa corporal (IMC), calculou-se o quociente do peso em quilogramas pelo quadrado da altura em metros $^{(9)}$. Foram utilizados os pontos de corte para critérios de baixo peso ( $<$ Percentil 5 ), eutrófico ou adequado $(\geq$ Percentil 5 e $<$ Percentil 85), sobrepeso ( $>$ Percentil 85 e $\leq 97$ ) e obesidade ( $>$ Percentil 97 e $\leq 99,9$ ), classificação do percentil de IMC para adolescentes do sexo feminino e masculino, com idade maior ou igual a 10 e menor de 20 anos, segundo curva de distribuição em percentis por sexo e idade, recomendados pelo $\operatorname{SISVAN}^{(9,10)}$ e $\mathrm{WHO}^{(11)}$.

Considerou-se praticante de exercício físico aquele que realiza qualquer modalidade no mínimo 2 vezes na semana, com duração de 60 minutos $^{(12)}$. Estipulou-se como presença de fator de risco para DCNT o consumo de: cigarro, álcool e drogas ilícitas (maconha, craque e/ou cocaína), independente da frequência, e de frutas e hortaliças inferior a uma vez ao dia. Enquadrou-se como hábito alimentar o consumo de alimentos gordurosos, como leite integral (ao menos uma vez por semana), carne com gordura aparente e guloseimas (chocolate, biscoitos recheados, doces, balas), em qualquer frequência, e refrigerantes por, no mínimo, cinco vezes por semana. O feijão foi o alimento escolhido para representar o grupo das leguminosas, sendo considerado hábito o consumo de, no mínimo, cinco vezes por semana.

Os dados coletados foram organizados em um banco de dados desenvolvido no Excel ${ }^{\circledR}$ e analisados no pacote estatístico BioEstat 5.3. Posteriormente, sucedeu-se a categorização e análise das variáveis quanto à freqüência (medidas antropométricas, experimentação de bebida alcoólica, fumar e/ou usar drogas ilícitas, perfil alimentar e prática de exercício físico). Realizaramse as associações entre variáveis categóricas (classificação de IMC, prática de exercício físico, tabagismo, etilismo, drogas ilícitas, marcadores de alimentos saudáveis e não saudáveis) separadamente entre sexo masculino e feminino, por meio do teste Qui-quadrado, nível de significância de $5 \%(\mathrm{p}<0,05)$.

O estudo obteve a aprovação da Comissão de Ética e Bioética da Faculdade de Imperatriz - Protocolo $\mathrm{n}^{\circ}$ 117/14, e autorização por escrito dos responsáveis dos alunos avaliados por meio do Termo de Consentimento Livre e esclarecido. 


\section{RESULTADOS}

Dos 473 adolescentes avaliados, 260 do sexo feminino e 213 do sexo masculino compuseram a amostra. A distribuição da idade dos escolares variou entre 10 e 17 anos com média 12,80 (IC: $12,68-12,93$ e DP $\pm 1,42$ ). A $7^{\circ}$ série apresentou a maior proporção de escolares, com 28,2\% (133). Considerando o estado nutricional dos pesquisados (desnutrição, sobrepeso, obesidade, eutrófico), a desnutrição foi superior à prevalência de obesidade, com 9,5\% (45). Porém, ao se considerar quadros de sobrepeso e obesidade (sobrepeso + obesidade), observa-se uma prevalência de 13,3\% (63) no total. A prevalência de 95,5\% (31) de sobrepeso e obesidade destacou-se no sexo masculino, assim como a desnutrição com 71,1 (32) (Tabela I).

Tabela I - Indicadores demográficos - idade, série e classificação do estado nutricional segundo percentil de massa corpórea (PIMC), por sexo, dos adolescentes matriculados em seis escolas públicas de Ensino Fundamental em Imperatriz, Maranhão, 2015.

\begin{tabular}{lccc}
\hline Variáveis & $\begin{array}{c}\text { Total } \\
\mathbf{n}(\%)\end{array}$ & $\begin{array}{c}\text { Feminino } \\
\mathbf{n}(\%)\end{array}$ & $\begin{array}{c}\text { Masculino } \\
\mathbf{n}(\mathbf{\%})\end{array}$ \\
\hline Idade (anos) & & & \\
10 & $17(3,6)$ & $9(53)$ & $8(47)$ \\
11 & $76(16,1)$ & $44(57,9)$ & $32(42,1)$ \\
12 & $116(24,5)$ & $65(56)$ & $51(44)$ \\
13 & $106(22,4)$ & $57(53,8)$ & $49(46,2)$ \\
14 & $104(22)$ & $61(58,7)$ & $43(41,3)$ \\
15 & $38(8)$ & $18(47,4)$ & $20(52,6)$ \\
16 & $15(3,2)$ & $6(40)$ & $9(60)$ \\
17 & $1(0,2)$ & $0(0)$ & $1(100)$ \\
Série & & & $52(47,7)$ \\
$6^{\circ}$ ano & $109(23)$ & $57(52,3)$ & $60(45,1)$ \\
$7^{\circ}$ ano & $133(28,2)$ & $73(54,9)$ & $55(44,7)$ \\
$8^{\circ}$ ano & $123(26)$ & $68(55,3)$ & $46(42,6)$ \\
$9^{\circ}$ ano & $108(22,8)$ & $62(57,4)$ & $32(71,1)$ \\
PIMC** & & & $24(53,3)$ \\
$<5$ (Desnutrição) & $45(9,5)$ & $13(28,9)$ & $67(43,5)$ \\
$5-15$ (Eutrófico) & $45(9,5)$ & $21(46,7)$ & $59(35,5)$ \\
$15-50$ (Eutrófico) & $154(32,6)$ & $87(56,5)$ & $23(51,1)$ \\
$50-85$ (Eutrófico) & $166(35,1)$ & $107(64,5)$ & $8(44,4)$ \\
$85-95$ (Sobrepeso) & $45(9,5)$ & $22(48,9)$ & \\
$>95$ (Obesidade) & $(3,8)$ & $10(55,6)$ &
\end{tabular}

n: Número de participantes incluídos e (\%) prevalência em 100\%; PIMC: Percentil do índice de massa corpórea

Dos alimentos julgados saudáveis e consumidos diariamente por mais de um entrevistado ( 5 dias ou mais por semana), destacaram-se arroz, feijão e frutas, 90,7\% (429), 76,7\% (363) e 67\% (217) respectivamente, enquanto que, iogurte, peixe e salada eram consumidos diariamente por apenas, respectivamente, 62,2\% (294), 36,8\% (174) e 63,6\% (301) (Tabela II). $\mathrm{O}$ consumo frequente de marcadores de alimentos saudáveis entre os pesquisados - arroz, feijão, peixe e salada - mostrouse superior no sexo feminino $(\mathrm{p}=0,0001)$. Ao mesmo tempo, os marcadores de hábitos alimentares não saudáveis, como consumo cotidiano de balinhas/doces $62,2 \%$ (294), bolacha recheada $52 \%$ (246) e refrigerante 46,9 (222) estão na rotina de todos os adolescentes, sem diferença entre os $\operatorname{sexos}(p=0,8397)$. Mais de um alimento saudável ou não saudável foi consumido simultaneamente pelos entrevistados. 
Tabela II - Indicadores de consumo de alimentos marcadores de padrões saudáveis e não saudáveis de alimentação segundo sexo, dos adolescentes matriculados em seis escolas públicas de Ensino Fundamental em Imperatriz, Maranhão, 2015.

\begin{tabular}{|c|c|c|c|c|c|c|c|}
\hline \multirow{2}{*}{ Indicadores } & \multicolumn{2}{|c|}{ Total } & \multicolumn{2}{|c|}{ Feminino } & \multicolumn{2}{|c|}{ Masculino } & \multirow{2}{*}{ Valor de $p^{*}$} \\
\hline & n“ & $\%$ & $\mathbf{n}$ & $\%$ & n & $\%$ & \\
\hline \multicolumn{8}{|l|}{ Consomem diariamente } \\
\hline Arroz** & 429 & 90,7 & 241 & 56,2 & 188 & 43,8 & 0,0001 \\
\hline Feijão** & 363 & 76,7 & 198 & 54,6 & 165 & 45,4 & \\
\hline Frutas** & 217 & 67 & 72 & 33,2 & 145 & 66,8 & \\
\hline Iogurte** & 294 & 62,2 & 167 & 56,8 & 127 & 43,2 & \\
\hline Peixe** & 174 & 36,8 & 105 & 60,3 & 69 & 39,7 & \\
\hline Salada** & 301 & 63,6 & 169 & 56,1 & 132 & 43,9 & \\
\hline Balinha ou doce*** & 294 & 62,2 & 167 & 56,8 & 127 & 43,2 & 0,8397 \\
\hline Bolacha recheada*** & 246 & 52 & 139 & 56,5 & 107 & 43,5 & \\
\hline Bolo $* * *$ & 198 & 41,9 & 119 & 60,1 & 79 & 39,9 & \\
\hline Refrigerante*** & 222 & 46,9 & 120 & 54,1 & 102 & 45,9 & \\
\hline Salgado*** & 150 & 31,7 & 83 & 55,3 & 67 & 44,7 & \\
\hline Sanduíche ou Hambúrguer*** & 134 & 28,3 & 72 & 53,7 & 62 & 46,3 & \\
\hline
\end{tabular}

*Valor de $p$ para o qui-quadrado da diferença entre adolescente do sexo feminino e masculino; ** Marcadores de alimentos saudáveis; *** Marcadores de alimentos não saudáveis." Número de entrevistados que consumiram os alimentos marcadores de padrão saudável e não saudável entre os 473 pesquisados.

A prática frequente de atividade física comparada aos hábitos sedentários revelou que 27,3\% (129) não realizavam nenhum tipo de exercício físico, em contraponto com 72,7\% (344), que praticavam alguma atividade física (Tabela III). Em relação à variável tempo de atividade física, o sexo feminino empenhou menor tempo em contraste com masculino, dos quais $59,1 \%$ (146) dedicavam cerca de uma hora ou mais, e 78,2\% (89) destes destinavam de 4 a 7 dias da semana para realizar suas atividades físicas.

Tabela III - Indicadores de atividade física e tempo gasto com os eletrônicos segundo sexo dos adolescentes matriculados em seis escolas públicas de Ensino Fundamental em Imperatriz, Maranhão, 2015.

\begin{tabular}{|c|c|c|c|c|c|c|c|}
\hline \multirow{2}{*}{ Variáveis } & \multicolumn{2}{|c|}{ Total } & \multicolumn{2}{|c|}{ Feminino } & \multicolumn{2}{|c|}{ Masculino } & \multirow{2}{*}{ valor de $p^{*}$} \\
\hline & $\mathbf{n}$ & $\%$ & n & $\%$ & n & $\%$ & \\
\hline \multicolumn{8}{|l|}{ Atividade Física** } \\
\hline Pratica & 344 & 72,7 & 161 & 46,8 & 183 & 53,2 & \multirow{2}{*}{0,0001} \\
\hline Não pratica & 129 & 27,3 & 99 & 76,7 & 30 & 23,3 & \\
\hline \multicolumn{8}{|c|}{ Tempo de Atividade Física } \\
\hline Menos de uma hora & 97 & 20,5 & 59 & 60,8 & 38 & 39,2 & \multirow[t]{3}{*}{0,0001} \\
\hline Uma hora ou mais & 247 & 52,2 & 101 & 40,9 & 146 & 59,1 & \\
\hline Não respondeu & 129 & 27,3 & 100 & 77,5 & 29 & 22,5 & \\
\hline \multicolumn{8}{|c|}{ Quantas vezes por semana pratica atividade física } \\
\hline 1 a 3 dias & 203 & 42,9 & 106 & 52,2 & 97 & 47,8 & \multirow[t]{4}{*}{0,0001} \\
\hline 4 a 5 dias & 48 & 10,2 & 20 & 41,7 & 28 & 58,3 & \\
\hline 6 a 7 dias & 96 & 20,3 & 35 & 36,5 & 61 & 63,5 & \\
\hline Não respondeu & 126 & 26,6 & 99 & 78,6 & 27 & 21,4 & \\
\hline \multicolumn{8}{|c|}{ Tempo gasto com eletrônicos $* * *$} \\
\hline 1 hora & 161 & 34 & 90 & 55,9 & 71 & 44,1 & \multirow[t]{5}{*}{0,5308} \\
\hline 2 horas & 112 & 23,7 & 55 & 49,1 & 57 & 50,9 & \\
\hline 3 a 4 horas & 47 & 9,9 & 24 & 51,1 & 23 & 48,9 & \\
\hline Mais de 4 horas & 141 & 29,8 & 84 & 59,6 & 57 & 40,4 & \\
\hline Não respondeu & 12 & 2,5 & 7 & 58,3 & 5 & 41,7 & \\
\hline
\end{tabular}

*Valor de p para o qui-quadrado da diferença entre adolescente do sexo feminino e masculino. ** Atividade física: prática de esporte e exercício físico. *** Eletrônicos: televisão, vídeo-game, computador e celular. 
O cômputo estatístico para tempo gasto com eletrônicos não diferiu entre os sexos, mesmo que elas destinassem mais de quatro horas aos eletrônicos. Quanto à exposição ao cigarro, drogas ilícitas e álcool, as adolescentes apresentaram maior prevalência, respectivamente 55,3\% (21), 58,3\% (7) e 57,4\% (70); no entanto, não ocorreu diferença significativa entre os sexos (Tabela IV).

Tabela IV- Indicadores de exposição ao cigarro, drogas ilícitas e álcool segundo sexo dos adolescentes matriculados na rede pública municipal de Ensino Fundamental em Imperatriz, Maranhão, 2015.

\begin{tabular}{|c|c|c|c|c|c|c|c|}
\hline \multirow{2}{*}{ Indicadores } & \multicolumn{2}{|c|}{ Total } & \multicolumn{2}{|c|}{ Feminino } & \multicolumn{2}{|c|}{ Masculino } & \multirow{2}{*}{ valor de $p^{*}$} \\
\hline & $\mathbf{n}$ & $\%$ & $\mathrm{n}$ & $\%$ & n & $\%$ & \\
\hline \multicolumn{8}{|l|}{ Cigarro } \\
\hline Experimentou & 38 & 8 & 21 & 55,3 & 17 & 44,7 & \multirow{2}{*}{0,8951} \\
\hline Não experimentou & 435 & 92 & 239 & 55 & 196 & 45 & \\
\hline \multicolumn{8}{|l|}{ Drogas ilícitas** } \\
\hline Experimentou & 12 & 2,5 & 7 & 58,3 & 5 & 41,7 & \multirow{2}{*}{0,9549} \\
\hline Não experimentou & 461 & 97,5 & 253 & 54,9 & 208 & 45,1 & \\
\hline \multicolumn{8}{|l|}{ Álcool } \\
\hline Experimentou & 122 & 25,8 & 70 & 57,4 & 52 & 42,6 & \multirow{2}{*}{0,6064} \\
\hline Não experimentou & 351 & 74,2 & 190 & 54,1 & 161 & 45,9 & \\
\hline
\end{tabular}

*Valor de p para o qui-quadrado da diferença entre adolescente do sexo feminino e masculino; ** Maconha, craque ou cocaína.

\section{DISCUSSÃO}

O presente estudo constatou o crescimento do quadro de sobrepeso, em especial entre o sexo feminino, corroborando com pesquisas similares que destacaram o crescimento do sobrepeso e obesidade ${ }^{(13,14)}$. O atual estudo foi realizado na segunda maior cidade do estado do Maranhão, onde políticas públicas têm sido intensificadas como o programa Mais IDH (Índice de Desenvolvimento Humano), cujo objetivo é inverter o cenário de extrema pobreza por meio de iniciativas e políticas públicas em áreas prioritárias, como Saúde, Educação, Cidadania, Infraestrutura, Assistência Social e Saneamento Básico. Não obstante essa realidade, a desnutrição ainda se destacou entre os escolares do sexo masculino incluídos no presente estudo, sustentando a necessidade de intensificação de ações do SPE em prevenir DCNT, cujos fatores de risco como obesidade, sobrepeso, inatividade física e rotina de consumo de alimentos não saudáveis prevalecem entre os adolescentes; no entanto, concomitante se convive com a morbidade moderna e doenças da pobreza.

Dos alimentos consumidos diariamente e enquadrados no grupo de alimentos saudáveis do presente estudo, o arroz (carboidrato complexo) e o feijão (minerais, fibras e proteínas) apresentaram maior proporção de consumo entre os adolescentes, dados parecidos com os da PenSE 2015(2), em que o feijão aparece como marcador alimentar saudável com maior proporção de consumo. Segundo o Guia Alimentar para a população brasileira, da Secretaria de Atenção à Saúde, a mistura de feijão com arroz aparece em quase todos os almoços selecionados, traduzindo a realidade alimentar da maioria dos brasileiros, que privilegiam alimentos in natura ou minimamente $\operatorname{processados}^{(15)}$.

Um estudo sobre a análise dos fatores associados ao excesso de peso em escolares ${ }^{(14)}$ salientou que o hábito de consumir doces e refrigerantes é mais frequente entre o sexo feminino, e a execução de atividade física, assim como a assiduidade semanal, é maior para o masculino. Todos os adolescentes entrevistados na presente pesquisa, no entanto, independente do sexo, admitiram passar mais de três horas assistindo televisão. O consumo de marcadores alimentares não saudáveis (balinhas ou doces, bolacha recheada e refrigerante) não diferiu entre os participantes, entretanto, hábitos saudáveis, como realizar atividade física, mais tempo na execução e maior frequência semanal, foram mais comuns entre o sexo masculino. Quanto ao tempo alocado em frente aos eletrônicos, os escolares, independente do sexo, passavam cerca de uma hora ou mais assistindo televisão, jogando videogame ou usando computador e celular. Fora o consumo de alimentos não saudáveis, as outras variáveis assemelharam-se ao estudo mencionado e a outros já realizados ${ }^{(16,17)}$.

Os resultados da Pesquisa Nacional de Saúde do Escolar (PeNSE, 2015)(2), contemplando os estudantes do $9^{\circ}$ ano do ensino fundamental nas capitais brasileiras evidenciou que o indicador de experimentação de cigarro para o sexo masculino (19,4\%) foi superior quando comparado ao feminino (17,4\%). Os dados revelaram também diferença na distribuição por sexo quanto à experimentação de álcool, com prevalência maior para as adolescentes (44,7\%). Quanto à experimentação de drogas ilícitas, os dados mostram que $9,0 \%$ dos escolares já usaram drogas ilícitas, sendo esse percentual de 9,5\% no sexo masculino e de $8,5 \%$ no feminino. No presente estudo, a experimentação de cigarro, drogas ilícitas e álcool não apresentou diferença entre os sexos, mas 
observou-se que a experimentação de bebidas alcoólicas teve maior proporção entre as adolescentes. Sendo este um importante fator de risco e avaliado como prevalente entre as mulheres, $\geq 18$ anos, integrantes da pesquisa do Vigitel, Ministério da Saúde, conduzida em $2011^{(18)}$. A OMS adotou uma meta de redução relativa de $10 \%$ no consumo per capita de álcool na população $\geq 15$ anos até $2025^{(19)}$. Medidas específicas dirigidas a essa população devem ser construídas, entre as quais se incluem ações regulatórias para o comércio e publicidade e propaganda, bem como o aumento da fiscalização, ambas previstas no Plano de Ações Estratégicas para o Enfrentamento das $\mathrm{DCNT}^{(7)}$.

O modelo de investigação apresentado no presente estudo mostrou-se factível e forneceu informações relevantes sobre a frequência de fatores de risco para doenças não transmissíveis, contudo algumas limitações devem ser consideradas, tais como: o uso de questionário pode aumentar a incidência de viés; há a possibilidade de que informações imprecisas tenham sido fornecidas pelos escolares, levados a negar ou afirmar algum hábito que viesse causar algum constrangimento; no decorrer do processamento dos dados e estudo da literatura viu-se a necessidade de pesquisar o comportamento dos pais referente às variáveis estudadas para serem associados aos costumes dos filhos e comparados com estudos existentes.

\section{CONCLUSÃO}

A população estudada apresenta distintos padrões de exposição a fatores de risco e proteção, e evidencia a necessidade de ações educativas no ambiente escolar, conduzidas pelos profissionais do programa municipal Saúde e Prevenção na Escola e demais gestores escolares no monitoramento dos fatores de risco de doenças crônicas não transmissíveis, como inatividade física, obesidade e sobrepeso, consumo de álcool, cigarros e drogas ilícitas, de forma emergencial, com intuito de permitir aos adolescentes, futuros adultos, uma vida saudável.

\section{AGRADECIMENTOS}

À Fundação de Amparo à Pesquisa e Desenvolvimento Científico do Maranhão (FAPEMA).

\section{REFERÊNCIAS}

1. World Heart Federation Urbanization and cardiovascular disease: Raising heart-healthy children in today's cities. Geneva: World Heart Federation; 2012.

2. IBGE. MINISTÉRIO DA SAÚDE (BRASIL). Pesquisa nacional de saúde do escolar : 2015 / IBGE, Coordenação de População e Indicadores Sociais. - Rio de Janeiro : IBGE, 2016.132 p.

3. Goldhaber-Fiebert JD, Rubinfeld RE, Bhattacharya J, Robinson TN, Wise PH. The utility of childhood and adolescent obesity assessment in relation to adult health. Med Decis Making. 2013;33:163---75

4. He F, Rodriguez-Colon S, Fernandez-Mendoza J, Vgontzas AN, Bixler EO, Berg A, et al. Abdominal obesity and metabolic syndrome burden in adolescents --- Penn State Children Cohort study. J Clin Densitom. 2015;18:30---6.

5. Beaglehole R, Bonita R, Horton R, Ezzati M, BhalaN, Amuyunzu-Nyamongo M, et al. Measuring progress on NCDs: one goal and five targets. Lancet. 2012 Oct;380(9850):1283-5.

6. Malta DC, Silva Junior JB. O Plano de Ações Estratégicas para o Enfrentamento das Doenças Crônicas Não Transmissíveis no Brasil e a definição da metas globais para o enfrentamento dessas doenças até 2025: uma revisão. Epidemiol. Serv. Saúde, Brasília. 2013; 22(1):151-164.

7. Brasil. Ministério da Saúde. Secretaria de Vigilância em Saúde. Departamento de Análise de Situação de Saúde. Plano de ações estratégicas para o enfrentamento das doenças crônicas não transmissíveis (DCNT) no Brasil 2011-2022 / Ministério da Saúde. Secretaria de Vigilância em Saúde. Departamento de Análise de Situação de Saúde. - Brasília : Ministério da Saúde, 2011. 148 p. : il. - (Série B. Textos Básicos de Saúde).

8. Ministério da Educação. Projeto Saúde e Prevenção nas Escolas (SPE). Secretária da Educação Básica, 2016. [acesso em 2017 Fev 24] Disponível em: http://portal.mec.gov.br/projeto-saude-e-prevencao-nas-escolas-spe

9. Sistema de Vigilância Alimentar e Nutricional. Orientações básicas para a coleta, processamento, análise de dados e informação em serviços de saúde [Andhressa Araújo Fagundes et al]. Brasília: Ministério da Saúde, 2004 [acesso em 2015 Jan 24] Disponível em: http://189.28.128.100/nutricao/docs/geral/orientacoes_basicas_sisvan.pdf.

10. Brasil. Ministério da Saúde. Secretaria de Atenção à Saúde. Departamento de Atenção Básica. Orientações para a coleta e análise de dados antropométricos em serviços de saúde : Norma Técnica do Sistema de Vigilância Alimentar e Nutricional - SISVAN / Ministério da Saúde, Secretaria de Atenção à Saúde, Departamento de Atenção Básica. - Brasília : Ministério da Saúde, 2011. 76 p. : il. - (Série G. Estatística e Informação em Saúde). 
11. World Health Organization. Preventing chronic diseases: a vital investment. Geneva: World Health Organization, 2006.

12. World Health Organization. Global Recommendations on Physical Activity for Health; 2011. [acesso em 2017 Fev 7 ]. Disponível em: http:/www.who.int/dietphysicalactivity/physical-activity-recommendations-5-17years.pdf

13. Vasconcellos MB, Anjos LA, Vasconcellos MTL. Estado nutricional e tempo de tela de escolares da Rede Pública de Ensino Fundamental de Niterói, Rio de Janeiro, Brasil. Cad Saúde Pública, Rio de Janeiro. 2013; 29(4): 713-722.

14. Pinto RP, Nunes AA, de Mello LM. Análise dos fatores associados ao excesso de peso em escolares. Rev Paul Pediatr. $2016 ; 34(4): 460-8$

15. Brasil. Ministério da Saúde. Secretaria de Atenção à Saúde. Departamento de Atenção Básica. Guia alimentar para a população brasileira / Ministério da Saúde, Secretaria de Atenção à Saúde, Departamento de Atenção Básica. - 2. ed. Brasília : Ministério da Saúde, 2014. 156 p. : il.

16. Vasconcellos MBD, Anjos LAD, Vasconcellos MTLD. Estado nutricional e tempo de tela de escolares da Rede Pública de Ensino Fundamental de Niterói, Rio de Janeiro, Brasil. Cad. Saúde Pública, Rio de Janeiro. 2013; 29(4):713-722.

17. Castro JAC, Nunes HEG, Silva DAS. Prevalência de obesidade abdominal em adolescentes:associação entre fatores sociodemográficos e estilo de vida. Rev Paul Pediatr. 2016; 34(3):343-351.

18. Ministério da Saúde (BR). Departamento de Análise de Situação de Saúde. Vigilância de fatores de risco e proteção para doenças crônicas por inquérito telefônico: Vigitel 2010. Brasília: Ministério da Saúde; 2011.

19. World Health Organization. Monitoring framework and targets for the prevention and control of NCDs: a comprehensive global monitoring framework, including indicators, and a set of voluntary global targets for the prevention and control of noncommunicabale diseases [Internet]. 2012 [citade 2012 set 6]. Geneva: World Health Organization: Available from: http://www.who.int/nmh/events/2012/ncd_discussion_paper/en/index.html

\section{Endereço do Primeiro Autor:}

Keise Adrielle Santos Pereira

Universidade Estadual da Região Tocantina do Maranhão - UEMA Sul

Laboratório Ciências da Saúde

Rua Godofredo Viana, 1300

Bairro: Centro

CEP 65900-100 - Imperatriz - MA - Brasil

E-mail: adriellekeise@gmail.com

\section{Endereço para Correspondência:}

Sheila Elke Araújo Nunes

Universidade Estadual da Região Tocantina do Maranhão - UEMA Sul

Laboratório Ciências da Saúde

Rua Godofredo Viana, 1300

Bairro: Centro

CEP 65900-100 - Imperatriz - MA - Brasil

E-mail: nunesearaujo@uol.com.br 